\title{
La douleur
}

La compréhension des mécanismes de la douleur a considérablement bénéficié des découvertes récentes des récepteurs morphiniques et des substances opioïdes endogènes. Dans un premier article, Jean-Marie Besson présente les données anatomophysiologiques essentielles sur ce sujet. Un second article traitera des mécanismes de contrôle et de leur implication dans le domaine thérapeutique.

\section{Jean-Marie Besson}

Directeur de recherche au Cnrs et de l'unité de neurophysiologie pharmacologique, Inserm UI $\sigma_{I}$.

\section{- glossaire •}

Cortex somesthésique primaire: aire corticule specifique de la sensation tactile dont l'organisation est strictement topique pour celle sensalion.

Decusser : croiser. passer de l'autre côti de la ligne médiane.

D.'sautonomie familiale : ma-

ladie autosomigue récessize, caracliriséc par un dysfonctionnement neurovégétalif el une insensibilite is la douleur associece is une diminution des fibres amyéliniques.

Rhizotomie postérieure: section des racines dorsales de la moelle épiniere.

Thalamus: faul partie du diencephale, comprend des amas de neurones constiluant diffërents noj'aux. Il constitue un important relais de differrentes modalites sensorielles zers le corlex cérébral.

\section{ADRESSE}

J.-M. Besson : Inserm U I61, 2, rue d'Alésia, 75014 Paris. e terme de douleur est utilisé pour désigner des expériences psychologiques variées, allant de celles évoquées dans des conditions expérimentales chez des individus sains, à celles observées dans des conditions pathologiques aiguës et chroniques. On distingue généralement trois composantes dans tout phénomène douloureux :

Une composante sensori-discriminative qui réfere à notre capacité d'analyser la nature (brûlure, piqûre, etc.), la localisation, l'intensité et la durée du stimulus; une composante motivationnelle qui affecte toute perception douloureuse d'un caractere désagréable; une troisième composante cognitive et évaluative impliquée dans les phénomènes d'anticipation, d'attention, de suggestion, d'expérience antérieure. Ces processus sont par ailleurs, capables d'agir sur la composante sensori-discriminative et/ou la composante motivationnelle par l'intermédiaire de différents systèmes de contrôles.

Nous considérerons uniquement ici les données essentielles relatives à la physiologie de la nociception. Ce terme découle des conclusions de Sherrington relatives aux stimulations capables de menacer l'intégrité de l'organisme. Chez l'animal, ces stimulations induisent diverses réponses réflexes et des comportements plus ou moins élaborés. Chez l'homme, la sensation induite par ces mêmes stimulations est souvent considérée comme une douleur aiguë, ce qui rend difficile la lálimi- tation d'une frontière précise entre concepts de nociception et de douleur. Néanmoins, on distingue classiquement la douleur aiguë, dont la finalité biologique d'alarme et de sauvegarde de l'intégrité de l'organisme la rapproche de la nociception et, la douleur chronique, liée ou non à une lésion du système nerveux central ou périphérique qui, par sa durée, constitue en plus une agression sévère affectant la personnalité de l'individu.

A partir des données principalement obtenues chez l'animal, nous envisagerons successivement dans ce premier article les mécanismes périphériques spinaux et centraux de la nociception.

\section{A la périphérie}

Il est généralement admis qu'il n'existe pas de structure spécifique histologiquement individualisée pouvant être qualifiée de récepteur nociceptif proprement dit, et que le message nociceptif résulte de la mise en jeu de terminaisons libres amyéliniques constituant des arborisations plexiformes dans les tissus cutanés et musculaires, ainsi que dans les parois des viscères.

Les messages nociceptifs sont ensuite véhiculés dans les nerfs par différentes fibres cataloguées selon leur diamètre et l'existence ou non d'une gaine de myéline. Les premières études psychophysiologiques effectuées chez l'homme ont montré que la sensation douloureuse résultait de la mise en jeu de fibres à conduction lente : les fibres $A \delta$ fai- 
blement myélinisées (diamètre 1 à 5 microns; vitesse de conduction : 4 à $30 \mathrm{~m} / \mathrm{s}$ ) et les fibres $C$ amyéliniques (diamètre 0,3 à 1,5 micron; vitesse de conduction : 0,4 à $2 \mathrm{~m} / \mathrm{s}$ ). L'existence de ces deux groupes de fibres afférentes fines peut expliquer la double douleur parf ois ressentie lors de l'application de stimulations cutanées intenses : les fibres A $\delta$ seraient alors responsables de la douleur rapide bien localisée et à type de piqûre, alors que l'activation des fibres $\mathrm{C}$ induirait la douleur tardive plus diffuse et à type de brûlure. Les techniques microphysiologiques, pratiquées principalement sur des nerfs cutanés, ont permis l'enregistrement unitaire de l'activité des fibres afférentes chez différentes espèces animales y compris chez l'homme où certaines équipes ont pu corréler leur activité avec la sensation perçue ( figure I). Schématiquement, deux groupes de nocicepteurs ont été décrits dans la peau : les mécanonocicepteurs $A \delta$ qui ne répondent qu'à de fortes stimulations mécaniques et les nocicepteurs polymodaux $C$ qui répondent à toutes les modalités des stimulations nociceptives (mécanique, thermique et chimique). En réalité la classification des nocicepteurs est beaucoup plus complexe et cette distinction ne doit pas être considérée de façon absolue car, en effet, comment expliquer la double douleur évoquée par des stimulations thermiques? D'ailleurs, chez l'homme, certaines études ont montré que des fibres $A \delta$ pouvaient se comporter comme des nocicepteurs polymodaux $C[1]$. Les nocicepteurs $A \delta$ et $C$ sont capables de coder l'intensité des stimulations, c'est-à-dire qu'ils accroissent leur réponse en fonction de l'intensité du stimulus appliqué. Chez l'homme, de nombreuses études ont permis de souligner le rôle majeur joué par les nocicepteurs polymodaux $C$ dans la détection et le codage de l'intensité de la douleur $[2,3]$. Leur seuil de réponse concorde avec celui de la sensation douloureuse dont, par ailleurs, l'intensité est bien corrélée avec le niveau d'activité de ces nocicepteurs. Lorsque l'on applique des stimulations répétées, ils sont le siège de phénomènes de sensibilisation qui se manifestent par une ou $m / s n^{\circ} 6$ octobre 85 plusieurs des modifications suivantes : diminution du seuil de réponse, augmentation des réponses, apparition d'une activité spontanée. Ces phénomènes peuvent être à l'origine des réactions d'hyperesthésie observées dans certaines conditions pathologiques chez l'homme.

Dans les muscles, un grand nombre de fibres $A \delta$ et $C$ sont des nocicepteurs polymodaux particulièrement excités par les substances algogènes et les stimulations thermiques. On ne peut cependant affirmer que toutes les fibres musculaires fines soient impliquées dans la nociception; en effet de par leur activation lors de la contraction musculaire, certaines pourraient correspondre à des "ergocepteurs" impliqués dans l'induction des réajustements circulatoires et respiratoires lors de l'exercice musculaire.

Il est actuellement difficile de savoir si la douleur d'origine viscérale résulte de la mise en jeu de nocicepteurs spécifiques ou de l'activation excessive de récepteurs qui déjà dans les conditions normales participent à la régulation réflexe de la fonction viscérale. Néanmoins, des fibres fines $A \delta$ ou $C$ activées par des stimulations qui, chez l'animal, déclenchent des réactions assimilables à la douleur, ont été mises en évidence dans le cœur, la plèvre et la cavité abdominale, la vésicule biliaire et les testicules. Certaines de ces fibres sont également excitées lors de la distension ou de la contraction modérée des viscères, et accroissent leur décharge avec l'augmentation de la stimulation. On ne peut donc pas les considérer uniquement comme des nocicepteurs et leur rôle reste à établir.

D'un point de vue général, les travaux électrophysiologiques mettant en évidence des nocicepteurs spécifiquement activés par des stimulations nociceptives sont à rapprocher des études morphologiques réalisées chez des malades présentant une insensibilité congénitale à la douleur, chez lesquels une absence ou une diminution du nombre de fibres myélinisées fines et/ou non myélinisées a été rapportée au niveau des nerfs périphériques [4]. Les mécanismes intimes responsables de la genèse des messages noci- ceptifs ne sont pas élucidés. Pourtant, même si l'éventualité d'une activation directe des terminaisons périphériques ne peut être écartée, de nombreuses données soulignent l'action excitatrice de certaines substances chimiques (histamine, sérotonine, kinines...) connues pour être libérées dans les tissus lésés et dans les différents exsudats inflammatoires. De plus, il est assez bien démontré que les prostaglandines également présentes dans les tissus lésés ou enflammés agissent en sensibilisant les terminaisons nerveuses à ces diverses substances algogènes.
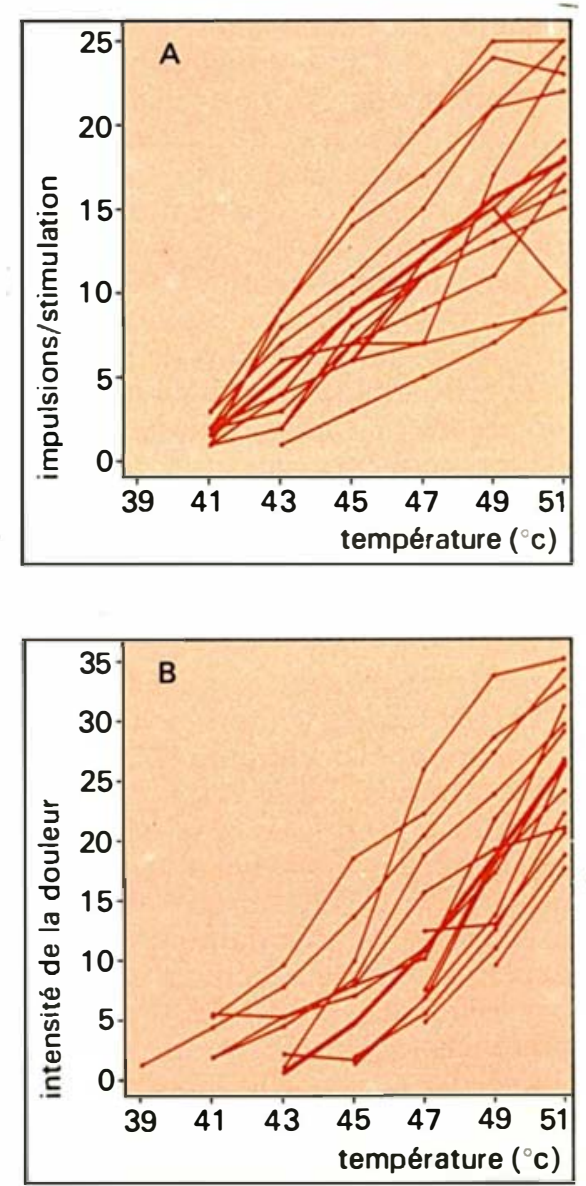

Figure I. Relation chez Phomme entre intensité de la décharge de nocicepteurs amyéliniques et intensité de la sensation de douleur évaluée par les mêmes sujets. A) Relation entre intensité de la décharge des fibres $C$ et intensité du stimulus thermique appliqué. B) Relation entre intensité de la douleur exprimée par les sujets et intensité de la stimulation thermique (d'après $(3 /)$. 
A cet effet, signalons que l'acide acétyl-salicyclique (Aspirine) inhibe la synthèse des prostaglandines [5] et réduit les réponses des nocicepteurs dans certaines conditions pathologiques ( figure 2).

\section{De la périphérie à la moelle}

Après leur trajet dans les nerfs périphériques, les fibres afférentes rejoignent le système nerveux central par les racines rachidiennes postérieures ou leur équivalent au niveau des nerfs crâniens. L'étude de leurs sites de terminaisons dans la substance grise médullaire [8] a bénéficié de nouvelles techniques telles que l'autoradiographie et le transport de la peroxydase du raifort, associés à l'examen en microscopie électronique. Les fibres fines $\mathrm{A} \delta$ et $\mathrm{C}$ se terminent de façon massive au niveau des couches superficielles (couche 1 , et couche 2 qui correspond à la substance gélatineuse) de la corne dorsale ipsilatérale. La stimulation des afférences nociceptives induit une excitation de certains neurones de la corne postérieure. La substance $P$ (peptide de 11 acides aminés) semble jouer un rôle important dans la transmission centrale des effets excitateurs des fibres fines. En effet ce peptide se trouve, comme le montrent les études tant biochimiques qu'immunohistochimiques, en forte concentration dans les couches superficielles [9], là où se terminent les afférences fines. De plus, la localisation de la substance $P$ dans ces dernières, est démontrée par la déplétion observée après rhizotomie postérieure* ou section d'un nerf périphérique. Enfin, ce peptide est libéré dans la moelle lors de la stimulation des fibres afférentes de fin diamètre [10] et il provoque (par injection locale) des effets excitateurs sur les neurones médullaires activés par des stimulations nociceptives [11]. Du point de vue clinique, une diminution du taux de substance $\mathrm{P}$ a été mise en évidence dans la substance gélatineuse chez des patients présentant une dysautonomie familiale* [12]. Ces données sont en faveur du rôle de médiateur que pourrait jouer la substance $P$ dans la nociception. Cependant, la
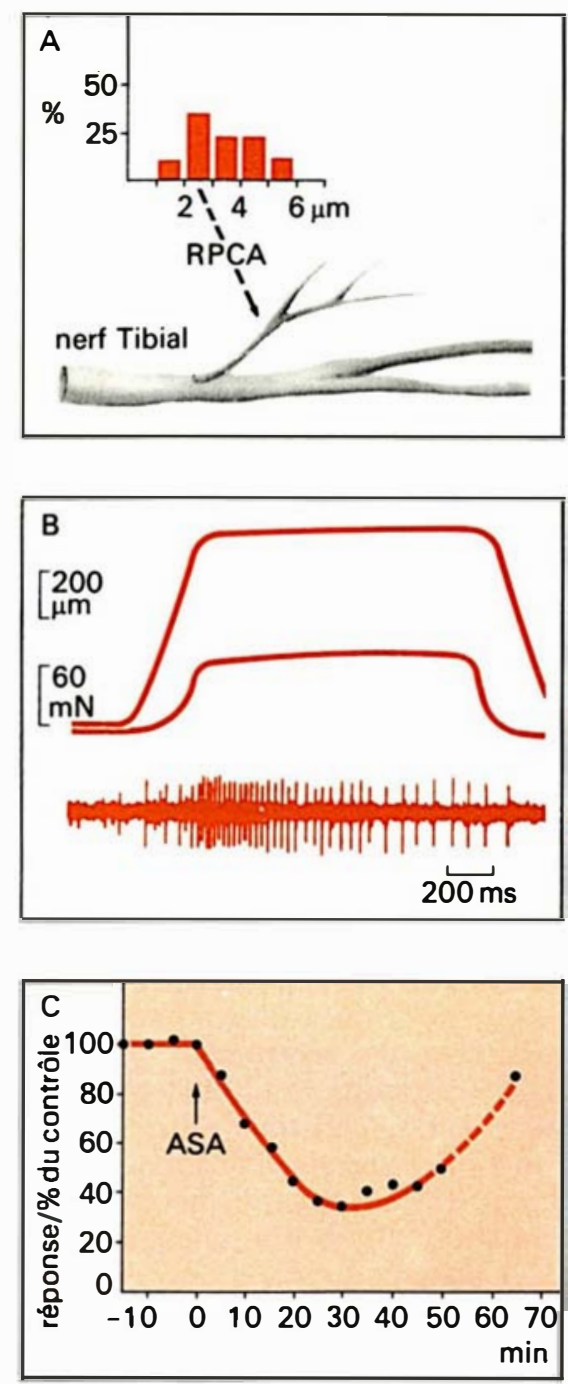

Figure 2. Démonstration électrophysiologique de l'effet périphérique de raspirine. A) Schéma du nerf tibial antérieur d'un rat, montrant le rameau primaire cutanéo-articulaire (RPCA) qui se divise lui-même en trois branches dont l'une contient les afférences innervant la capsule de la cheville. L'histogramme indique la proportion des fibres contenues dans ce rameau selon leur diamètre. B) Réponse d'une fibre $d u$ $R P C A$, chez un rat arthritique, lors de l'application d'une stimulation mécanique contrôlée. Le tracé supérieur figure lindentation (en micromètres), le tracé du milieu indique la force (en millinewton) qui est ainsi développée. C) Une réponse similaire à celle présentée en $B$ est déprimée par laspirine (ASA) directement appliquée au niveau de la capsule de la cheville enflammée chez un rat arthritique (d'après $[6,7]$ modifié). substance $P$ n'est pas l'unique peptide présent dans les petites cellules du ganglion rachidien puisque de la somatostatine et de la cholecystokinine, entre autres, ont été localisées dans ces cellules, et également d'ailleurs dans des neurones des couches superficielles elles-mêmes. De plus, certaines fibres afférentes peuvent contenir plus d'un peptide. Enfin, du point de vue électrophysiologique, les effets de la substance $P$ (injectée localement) sur les neurones spinaux activés par des stimulations nociceptives sont loin d'être univoques. Il s'agit d'un problème complexe et il est vraisemblable que certains rôles physiologiques actuellement attribués à la substance $P$ pourraient en fait être dus à d'autres peptides tels que les Tachykinines [13]. Quoiqu'il en soit, cet aspect neurochimique des mécanismes périphériques de la nociception ouvre de nouveaux horizons dans l'exploration des neuropathies où les troubles de la sensibilité douloureuse dominent, et permet d'autre part d'espérer la mise au point de thérapeutiques qui agiraient sélectivement sur la transmission périphérique des messages nociceptifs.

Dans la corne dorsale de la moelle, deux principaux groupes de cellules $[14,15]$ sont activés par la mise en jeu des fibres fines: (a) les neurones nociceptifs non spécifiques qui répondent à la fois à des stimulations mécaniques légères et aux stimulations nociceptives mécaniques et thermiques; ils possèdent la propriété d'accroître leur décharge en fonction de l'intensité du stimulus ( figure $3 A$ ); $(b)$ les neurones nociceptifs spécifiques qui ne sont excités que par des stimulations mécaniques et/ou thermiques nociceptives ( figure $3 B$ ). Ces neurones (surtout ceux du premier groupe) sont en plus activés par des stimulations viscérales appliquées au niveau du cœur, de la vessie, du testicule, ou par l'injection de substances algogènes dans l'artère mésentérique inférieure et par des stimulations musculaires intenses. L'existence de convergences viscérosomatiques va dans le sens de la théorie de la "projection convergente " [17], avancée pour expliquer le mécanisme des douleurs 

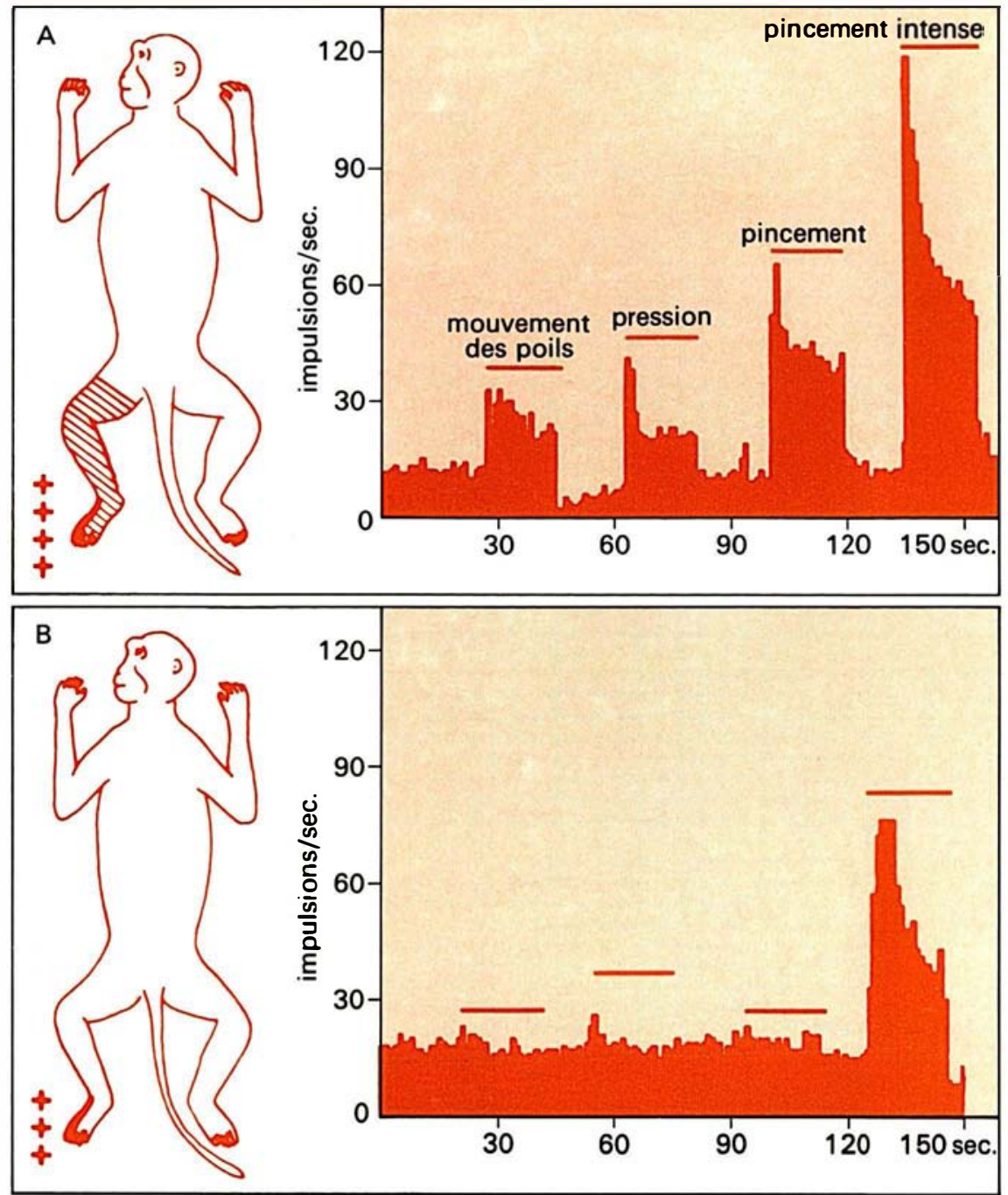

Figure 3. Caractéristiques des deux principaux types de neurones nociceptifs rencontrés au niveau de la corne dorsale de la moelle. A) Neurone nociceptif non spécifique qui accentue sa décharge en fonction de lintensité du stimulus. B) Neurone nociceptif spécifique. Les champs récepteurs de ces neurones sont indiqués sur la partie gauche de la figure (d'après [I6] modifié).

projetées. Selon cette théorie, ces douleurs seraient liées à la convergence de messages nociceptifs cutanés et viscéraux sur une population de neurones spinaux qui transmettent l'information aux centres supramédullaires. Dans les conditions habituelles, ceux-ci seraient surtout activés par les nocicepteurs des téguments; si dans des conditions non physiologiques, ils étaient activés par des nocicepteurs viscéraux, l'information nociceptive serait alors interprétée comme provenant des territoires cutanés qui en sont habituellement l'origine. Par exemple l'angine de poitrine se traduit fréquemment par une douleur $m / s n^{\circ} 6$ octobre 85

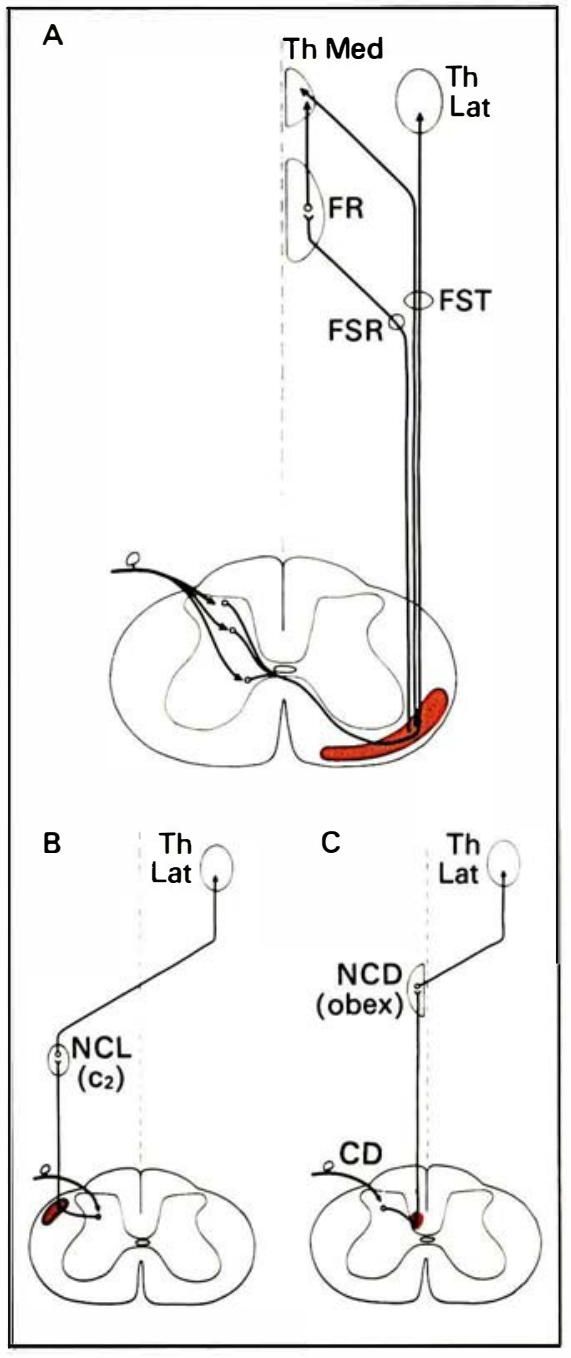

Figure 4. Représentation schématique des principaux faisceaux ascendants. A) Faisceau spinoréticulaire (FSR) et faisceau spinothalamique (FST) cheminant dans le cordon antérolatéral. FR : formation réticulaire; Th Med : thalamus médian; Th Lat : thalamus latéral. B) Faisceau spinocervicothalamique cheminant dans le cordon dorsolatéral et relayant dans le noyau cervical latéral (NCL) avant de se projeter au niveau du thalamus latéral. C) Fibres postsynaptiques des colonnes dorsales (CD) atteignant les noyaux des colonnes dorsales (NCD) (d'après [15] modifié).

que les messages nociceptifs véhiculés par ces fibres atteignent les structures supraspinales à partir des relais respectivement localisés dans le noyau cervical latéral et les noyaux des colonnes dorsales (comme indiqué figure $4 B, C$ ). 


\section{REFERENCES}

I. Adriaensen $\mathrm{H}$, Gybels J, Handwerker $\mathrm{HO}$ Van Hees J. Response properties of thin myelinated (A $\delta$ ) fibers in human skin nerves. 7 Neurophysiol 1984; 49 : 111-22.

2. Gybels J, Handwerker HO, Van Hees J. Comparison between the discharge of human nociceptive nerve fibers and the subject's rating of his sensation. 7 Physiol (Lond) 1979; 292 : 193-206.

3. Torebjork HE, Lamotte RH, Robinson CJ. Peripheral neural correlates of magnitude of cutaneous pain and hyperalgesia: simultaneous recordings in humans of sensory judgements of pain and evoked responses in nociceptors with $C$ fibers. 7 Neurophysiol 1984; 51 : 325-39.

4. Gocbel HH, Veit S, Dyck PJ. Confirmation of virtual unmyelinated fiber absence in heredity sensory neuropathy type IV. 7 Neuropath Exp Neurol 1980; $39: 670-5$

5. Vane JR. Inhibition of prostaglandin synthesis as a mechanism of action for aspirin like drugs. Nature 1971; 231 : 232-5.

6. Guilbaud G, Iggo A, Tegner R. Sensory receptors in ankle joint capsules of normal and arthritic rats. Exp Brain Res 1985; 58 : 29-40.

7. Guilbaud G, Iggo A. The effects of aspirin on joint capsule mecanoreceptors in rats with polyarthritis. Exp Brain Res. In press.

8. Perl ER. Characterization of nociceptors and their activation of neurons in the superficial dorsal horn. First steps for the sensation of Pain In: Kruger L, Liebeskind JC, eds. Neuronal Mechanisms of Pain. Advances in Pain Research. vol. 6. New York: Raven Press, 1984 : 23-51.

9. Hokf elt T, Kellerth JO, Nilsson G, Pernow B Experimental immunohistochemical studies on the localization and distribution of substance $P$ in cat primary sensory neurons. Brain Res 1975; $100: 235-52$.

10. Yaksh TL, Jessell TM, Gamse R, Mudge $A W$, Lecman SE. Intrathecal morphine inhibits substance $P$ release from mammalian spinal cord in vivo. Nature 1980; $286: 155-7$.

I 1. Henry JL. Effects of substance P on functionnally identified units in cat spinal cord. Brain Res 1976; $114:$ :439-51.

12. Pearson J, Brandeis I, Cuello AC. Depletion of substance $P$ containing axons in substantia gelatinosa of patients with diminished pain sensitivity. Nature 1982; $295: 6 \mathrm{I}-63$
En revanche, des observations cliniques chez l'homme et des données électrophysiologiques chez l'animal démontrent indiscutablement que la plus grande partie des fibres ascendantes qui mettent en jeu les structures supraspinales responsables de la sensation douloureuse, proviennent de neurones dont les axones décussent* au niveau médullaire et empruntent le quadrant controlatéral de la moelle (figure $4 A$ ). La section de ce dernier (cordotomie antérolatérale) est encore fréquemment employée pour le traitement des douleurs rebelles notamment d'origine néoplasique. Le faisceau spinothalamique est fréquemment assimilé a tort au cordon antérolatéral, en réalité il contient d'autres voies
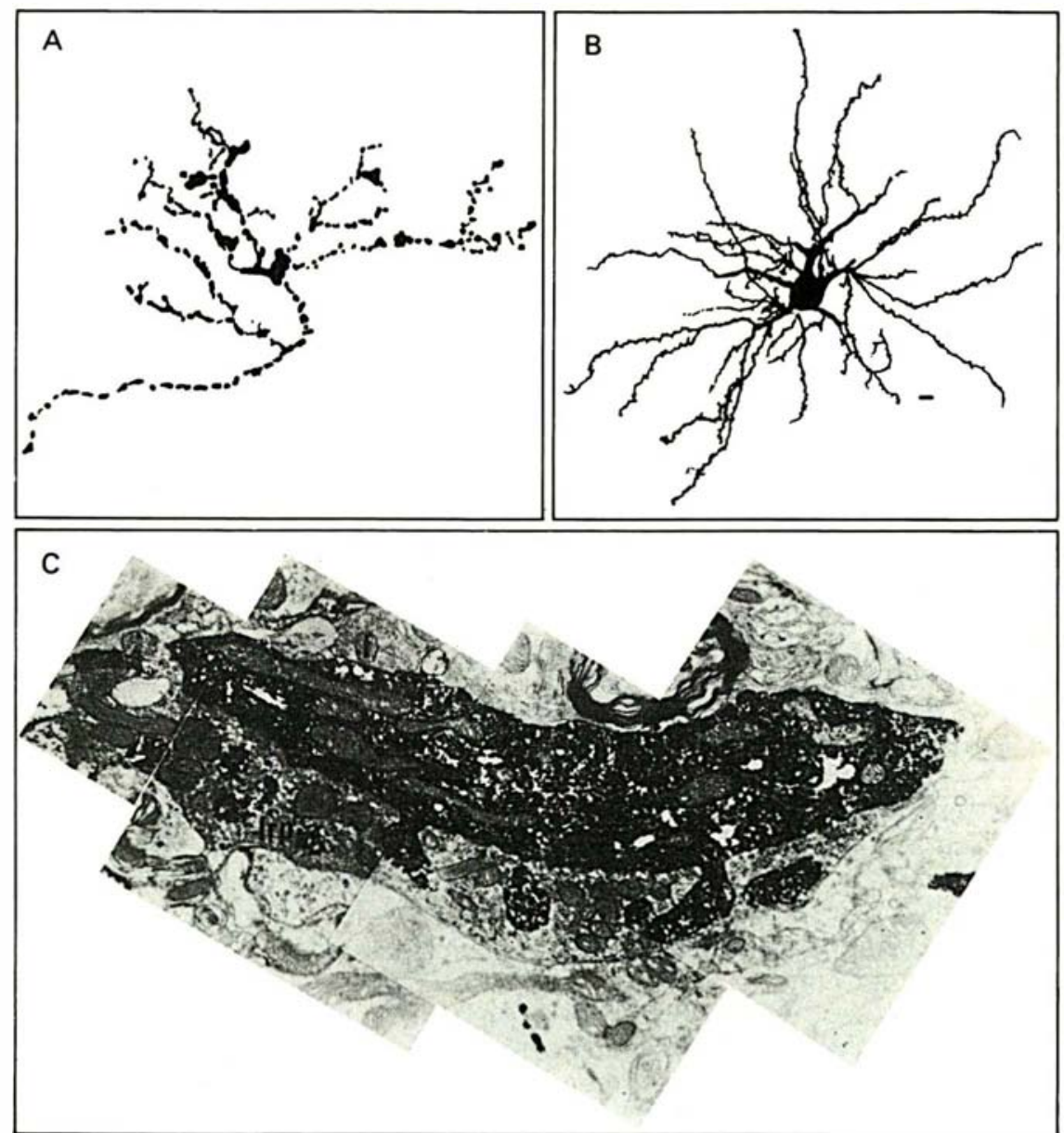

Figure 5. Exemples d'études anatomiques permettant de localiser des neurones thalamiques recevant des messages nociceptifs. A) Visualisation d'une fibre spinothalamique marquée par le transport antérograde de peroxydase du raifort couplée à une lectine. B) Reconstruction, après injection intracellulaire de peroxydase $d u$ raifort, d'un neurone thalamique répondant à des stimulations nociceptives. C) Terminaison (probablement spinothalamique) recevant de multiples contacts synaptiques avec un des dendrites primaires du neurone présenté en $B[19,20]$.

$m / s n^{\circ} 6$ octobre 8 
ascendantes notamment le faisceau spinoréticulaire. On ne peut donc attribuer une fonction univoque à un faisceau donné; néanmoins, par son pourcentage appréciable de neurones nociceptifs et par l'importance des convergences cutanées, viscérales et musculaires sur ces neurones, le faisceau spinothalamique occupe une place privilégiée dans la transmission des messages nociceptifs chez les primates [18]. Cependant le développement récent de techniques anatomiques extrêmement performantes, a permis de mettre en évidence la complexité de l'organisation des voies ascendantes. A titre d'exemple, mentionnons qu'une seule fibre ascendante peut se projeter à la fois aux niveaux réticulaire et thalamique. L'analyse quantitative de ces collatéralisations permettra de juger plus précisément de l'importance respective des faisceaux ascendants.

\section{Au niveau supraspinal}

Les recherches se heurtent à des difficultés de divers ordres : multiplicité des faisceaux ascendants, différences interspécifiques, problèmes d'éthique nécessitant l'utilisation de préparations anesthésiées... Il ne fait aucun doute que les stimulations nociceptives mettent en jeu de nombreuses structures et les désillusions de la neurochirurgie stéréotaxique soulignent bien qu'il n'existe pas une région privilégiée dont la destruction permettrait de soulager les malades.

Les régions où se terminent les axones spinothalamiques ( figure 5) ont été particulièrement étudiées. De nombreux neurones répondant à des stimulations nociceptives ont été mis en évidence dans le complexe ventrobasal du thalamus* $[21,22]$ où se projettent également les afférences tactiles provenant des noyaux des colonnes dorsales (noyaux de Goll et Burdach).

Le seuil de réponses de ces neurones à la stimulation thermique se situe clairement dans la gamme des températures nociceptives $\left(>43^{\circ} \mathrm{C}\right)$. Certains d'entre eux (figure 6) codent l'intensité de la stimulation, sa surface et parfois sa durée. En raison de ces caractéristiques électrophysiologiques, ils sont vrai-
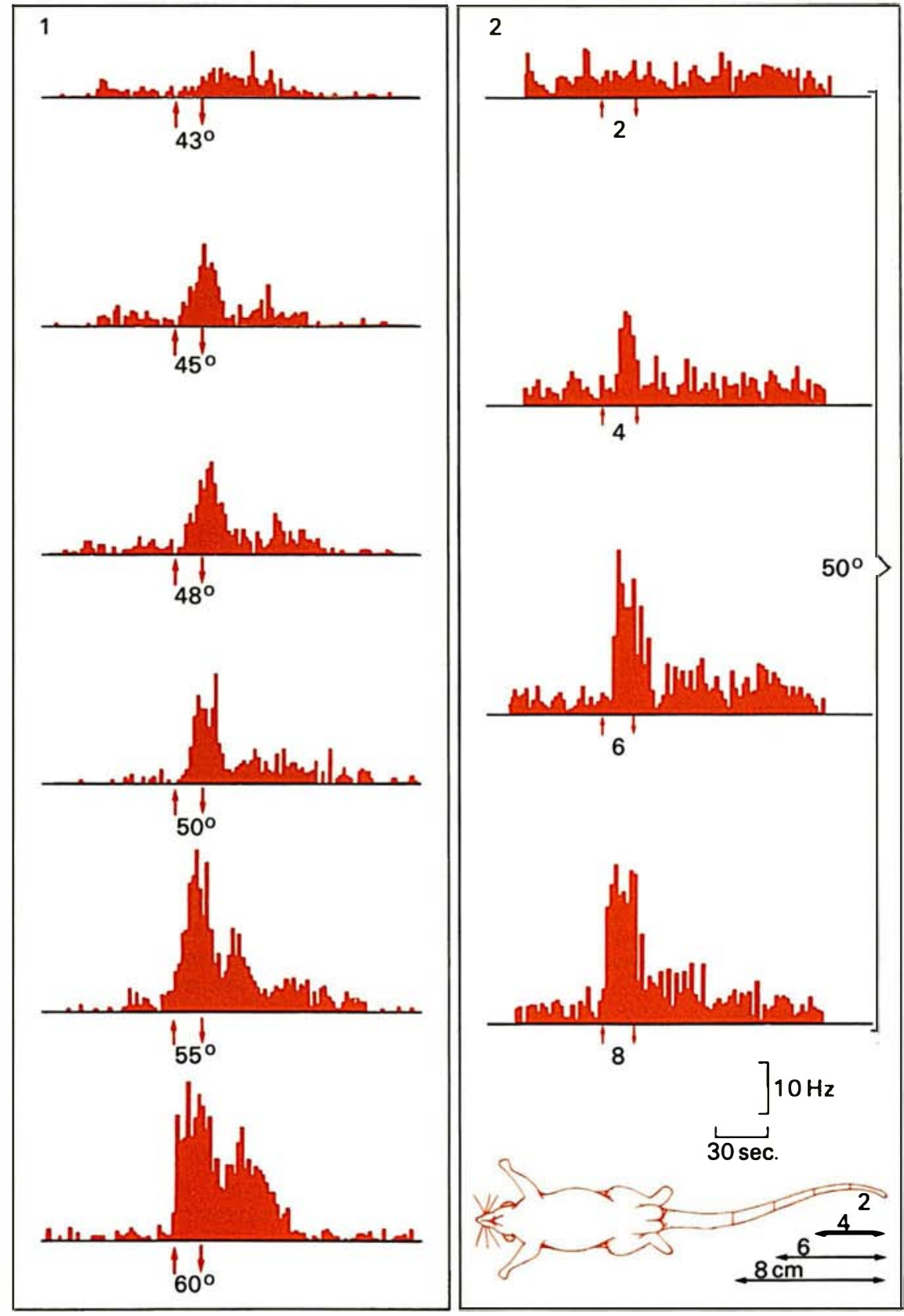

Figure 6. Réponses de neurones thalamiques (complexe ventrobasal du rat) à des stimulations thermiques nociceptives appliquées au champ récepteur localisé dans la queue. En $I$, le neurone est capable de coder l'intensité de la stimulation thermique; en 2 , pour une même intensité de stimulation, la réponse du neurone s'accroît en fonction de la surface de stimulation [23]. 


\section{RÉFÉRENCES}

13. Jessell TM, Womack MD. Substance $P$ and the novel mammalian tachykinins: A diversity of receptors and cellular actions. TINS 1985; 8 : 43-5.

14. Besson JM, Guilbaud G, Abdelmoumene M, Chaouch A. Physiologie de la nociception. 7 Physiol (Paris) 1982; $78:$ 7-107.

15. Dubner R, Bennett GJ. Spinal and trigeminal mechanisms of nociception. Ann Rev Neurosci 1983; $6: 381-418$.

16. Willis WD. Ascending pathways from the dorsal horn. in: Brown AG, Rethely M, eds. $S$ pinal cord sensation. Edinburgh: Scottish Academic Press, 1981 : 169-78.

17. Ruch TC. Visceral sensation and referred pain. In: Fulton JF, ed. Howell's Textbook of Physiology. Philadelphia: Saunders, $1949: 360-74$

18. Willis WD. The spinothalamic tract. In: Rosenberg RN, ed. The clinical neurosciences, vol. 5. Neurobiology. New York: Churchill Livingstone, $1983: 325-56$

19. Peschanski M, Lee CL, Ralston HJ. The structural organization of the ventro-basal complex of the rat as revealed by an analysis of physiologically characterized neurons injected intracellularly with horseradish peroxydase. Brain Res 1984; 297 : 63-74.

20. Peschanski M. Trigeminal afferent to the diencephalon in the rat. Neuroscience $1984 ; 12$ : 465-87.

21. Peschanski M, Guilbaud G, Gautron M, Besson JM. Encoding of noxious heat messages in neurons of the ventrobasal complex of the rat. Brain Res 1980; 197 : 401-13.

22. Kenshalo DR Jr, Giesler GJ Jr, Leonard B, Willis WD. Responses of neurons in primate ventral posterior lateral nucleus to noxious stimuli. 7 Neurophysiol 1980; 43 : 1594-1614.

23. Guilbaud G, Peschanski M, Kayser V, Benoist JM. Properties of nociceptive neurons in the thalamic ventrobasal complex in mammalian and arthritic rats. In: Rowe M, Willis WD, eds. Development, organization and processing in somatosensory pathways. New York: Liss AR, 1985: $383-91$.

24. Lamour Y, Willer JC, Guilbaud G. Rat somatosensory (SmI Cortex): Characteristics of neuronal responses to noxious stimulation and comparison with responses to non-noxious stimulation. Exp Brain Res 1983; 49 : 39-45.

25. Kenshalo DR Jr, Isensee O. Responses of primate SI cortical neurons to noxious stimuli. 7 Neurophysiol 1983; 50 : 1479-96.

26. Guilbaud G, Peschanski M, Besson JM. Experimental data related to nociception and pain at the supraspinal level. In: Wall PD, Melzack $R$, eds. Textbook of Pain. Edinburgh: Churchill Livingstone, $1984: 110-8$ semblablement impliqués dans la composante sensoridiscriminative de la douleur, ce qui est en bon accord avec le fait que ces neurones thalamiques nociceptifs transmettent l'information au cortex somesthésique primaire* $[24,25]$. Il est donc remarquable que, malgré la complexité des divers substrats neuronaux impliqués dans la nociception, l'on retrouve aux niveaux thalamique et cortical, des caractéristiques de réponses neuronales précédemment décrites pour des nocicepteurs périphériques et des neurones médullaires, tel que le codage de l'intensité du stimulus.

Des neurones nociceptifs ont été également mis en évidence aux niveaux d'autres régions (bulbe ponto-mésencéphalique, thalamus médian) où se terminent les fibres empruntant les voies spinoréticulaire et spinoréticulothalamique. Compte tenu de leurs caractéristiques fonctionnelles : champs récepteurs diffus et mal délimités, absence de codage, variabilité des réponses d'une stimulation à l'autre, il semble difficile d'attribuer à ces neurones un rôle spécifique dans la transmission et l'intégration des messages nociceptifs, tout au moins pour l'aspect sensoridiscriminatif. En revanche, en raison de leur projection sur les aires corticales motrices et prémotrices, ils pourraient intervenir dans l'élaboration des réactions motrices ou émotionnelles et dans les mécanismes d'éveil qui contribuent à la mise en jeu des systèmes de défense de l'organisme face à une agression nociceptive [26]. De plus, certaines connexions avec d'autres régions de l'encéphale (hypothalamus, rhinencéphale, autres aires corticales...) suggèrent leur implication dans les composantes motivationnelle, cognitive et évaluative de la douleur.

Tel que nous venons de le décrire, le circuit de la douleur apparaît comme un système rigide permettant la transmission des messages nociceptifs de la périphérie jusqu'aux centres superieurs de l'encéphale. En réalité, aux différents niveaux de ce circuit, le transf ert de l'information est constamment modulé par différents systèmes de contrôles dont on envisagera le rôle dans le prochain article

\section{Summary}

The transmission of nociceptive messages is initiated via activation of free nerve endings of mechano, thermo and chemosensitive peripheral afferent fibers of the skin, muscle and viscera. In general, the mechanonociceptors afferents are thinly myelinated A delta fibers; the polymodal nociceptors are unmyelinated $\mathrm{C}$ fibers. The peripheral afferents code for intensity of the stimulus and in humans the discharge of the $C$ nociceptors correlates well with the intersity of perceived pain. These afferents contain a variety of neuropeptides which have been implicated in the transmission of nociceptive information to the two main classes of dorsal horn neurons, nociceptive specific and wide dynamic intensity range neurons. Axons of the second order nociceptive neurons project rostrally, predominantly via the contralateral spinothalamic and spinoreticular tracts. Nociceptive neurons of the thalamus (ventrobasal complex) and cortex (primary somatosensory area), code for intensity of the noxious stimulus and have been implicated in the discriminative aspects of pain. The affective component of pain is attributed to simultaneous activation of ascending (reticular/limbic) systems.

\section{TIRES A PART}

J.-M. Besson : Inserm U I6I, 2, rue d'Alésia, 75014 Paris. 